\title{
Ferulic acid exhibits anti-inflammatory effects by inducing autophagy and blocking NLRP3 inflammasome activation
}

\author{
Yongjuan $\mathrm{Liu}^{1} \cdot \mathrm{Lu} \mathrm{Shi}^{2} \cdot$ Wenhong $\mathrm{Qiu}^{3} \cdot$ Yingying Shi $^{3}$ \\ Accepted: 29 December 2021 / Published online: 10 January 2022 \\ (c) The Author(s) under exclusive licence to The Korean Society of Toxicogenomics and Toxicoproteomics 2022
}

\begin{abstract}
Background Inflammation is involved in the healing process; however, when inflammation is overactivated, multiple diseases can occur. The continued discovery of new anti-inflammatory drugs is crucial in the treatment of inflammation-linked diseases.

Objectives Ferulic acid (FA), a precursor necessary for lignan synthesis, is widely distributed in plant-based whole foods and is a strong antioxidant. However, the effect of FA on the expression level of inflammatory factors in macrophages has not been fully clarified. The current study aimed to explore the anti-inflammatory effect and mechanism of ferulic acid.

Results The results showed that THP-1 cells were induced to differentiate into macrophages by Phorbol-12-myristate13-acetate (PMA), and THP-1-derived macrophages were stimulated by LPS to establish an inflammatory cell model. Compared with the control group, low $\left(5 \mu \mathrm{mol} \cdot \mathrm{mL}^{-1}\right)$, medium $\left(10 \mu \mathrm{mol} \cdot \mathrm{mL}^{-1}\right)$, and high $\left(20 \mu \mathrm{mol} \cdot \mathrm{mL}^{-1}\right)$ concentration ferulic acid groups have decreased cell viability and increased apoptosis rate in a dose-dependent manner. FA reduced the transcriptional levels of Interleukin-1 $\beta$ (IL-1 $\beta$ ), Interleukin-6 (IL-6) and tumor necrosis factor $\alpha$ (TNF- $\alpha$ ). Importantly, FAinduced autophagy and inhibited NLRP3 inflammasome activation. 3-MA (a widely used autophagy inhibitor) enhanced the secretion of TNF- $\alpha$, IL- 6 and IL-1 $\beta$. Moreover, autophagy inhibition by 3-MA resulted in increased proteins expression associated with NLRP3 inflammasome signaling pathway. Besides, the inhibition of inflammasome activation by MCC950 reduced the expression of TNF- $\alpha$, IL- 6 and IL- $1 \beta$.

Conclusion It is concluded that FA enhanced autophagy, inhibited the activation of NLRP3 inflammasome and reduced the expression and release of inflammatory factors.
\end{abstract}

Keywords Ferulic acid $\cdot$ Macrophage $\cdot$ Autophagy $\cdot$ NLRP3 inflammasome $\cdot$ Inflammatory factor

\section{Introduction}

Inflammation is vital to defend against tissue injury, infection or toxic agents and involves recruitment of phagocytes (macrophages and neutrophils) and the secretion of various cytokines (Medzhitov 2008). During an inflammatory process, macrophages are one of the most important phagocytes,

Yingying Shi

shiyingyinga@126.com

1 Department of Central Laboratory, Affiliated Lianyungang Hospital of Xuzhou Medical University, Lianyungang 222002, Jiangsu, China

2 Department of Pharmacy, School of Medicine, Jianghan University, Wuhan 430056, Hubei, China

3 Department of Immunology, School of Medicine, Jianghan University, Wuhan 430056, Hubei, China which can participate in the development of a variety of diseases by secreting a large number of cytokines, such as TNF- $\alpha$, IL-6 and IL-1 $\beta$ (Medzhitov 2010). Such inflammatory mediators can act on both immune cells and nonimmune cells to protect the body from harmful substances and pathogens by inducing an immune response (Medzhitov 2008). Persistent inflammation has been associated with several diseases, such as cancers, arteriosclerosis, rheumatoid arthritis, asthma and Alzheimer's disease (Back et al. 2019; DiDonato et al. 2012; Glass et al. 2010).

THP-1 is a human monocytic cell line that commonly used in the laboratory and can differentiate into macrophages by inducing with phorbol 12-myristate 13 acetate (PMA) (Lund et al. 2016; Biriken et al. 2018). Macrophages express various pathogen recognition receptors, including Toll-like receptors (TLRs) (Gruber and Leifer 2020). Lipopolysaccharide (LPS) can be recognized by TLR-4 and further activates 
the specific signaling pathways downstream of TLR-4, leading to the activation of NF- $\mathrm{KB}$ and proinflammatory genes expression (Liu et al. 2017).

Inflammasomes are widely distributed cytosolic sensors which can sense metabolic imbalances or pathogen infections and trigger the release of proinflammatory cytokines (Guo et al. 2015). Currently, one of the most well-studied inflammasomes is NOD-like receptor-family pyrin domaincontaining 3 (NLRP3) inflammasome (Broz and Dixit 2016). According to research findings, the full activation of NLRP3 inflammasomes requires two signals (Franchi et al. 2012). The first signal involves the interaction between TLRs and their ligands, the activation of NF- $\mathrm{KB}$ and the synthesis of pro-IL-1 $\beta$ and NLRP3 (Franchi et al. 2012). The second stimulus leads to caspase- 1 autoactivation, the cleavage of pro-IL-1 $\beta$, and the release of mature IL- $1 \beta$ (Franchi et al. 2012). Appropriate concentration of lipopolysaccharide has been confirmed to provide two activation signals in human monocytes and macrophages and led to IL- $1 \beta$ secretion (Petes et al. 2017; Tang et al. 2019; Zhao et al. 2019). As a multifunctional inflammatory factor, IL- $1 \beta$ performs both immunity and anti-infective functions by activating the expression of other immune genes and recruiting lymphocytes to the site of infection (Dinarello 2011).

Autophagy plays a critical role in maintaining cell homeostasis by means of circulating materials and energy inside the cells in an environment that is not conductive to cell growth (Glick et al. 2010). The NLRP3 inflammasome is an important part of the innate immune system (Guo et al. 2015). Numerous studies have shown that there is a complex regulatory relationship between autophagy and inflammasome (Cao et al. 2019). Autophagy has been found to inhibit NLRP3 inflammasome activation in some cases and activate NLRP3 inflammasome activation in other cases (Cao et al. 2019). Meanwhile, NLRP3 inflammasome has been reported to affect autophagy (Cao et al. 2019). In this study, the complex regulatory relationship between autophagy and NLRP3 inflammasome was further clarified.

Ferulic acid (FA) is a polyphenolic compound that can be found in a variety of plants, for example grains, vegetables and fruits (de Oliveira et al. 2015). FA has been confirmed to have various biological activities, such as antimicrobial, anticarcinogenic, antioxidant, hepatoprotective, and vasodilatory actions (Amic et al. 2020; Zhang et al. 2019; Zhou et al. 2020). FA has been reported to have anti-inflammatory effects in several studies. Ferulic acid has been reported to inhibit bovine endometrial epithelial cells against LPSinduced inflammation via suppressing NK-kappa B and MAPK pathway (Wu et al. 2021). Ferulic acid also ameliorates hepatic inflammation and fibrotic liver injury by inhibiting PTP1B activity and subsequent promoting AMPK phosphorylation (Wu et al. 2021). Meanwhile, FA has been confirmed to induce autophagy and alleviates lipotoxicityinduced hepatocellular death through the SIRT1-regulated autophagy pathway (Xu et al. 2021). Ferulic acid protects cardiomyocytes from TNF-alpha/cycloheximide-induced apoptosis by regulating autophagy ( $\mathrm{Li}$ et al. 2020). FA mitigated oxygen-glucose deprivation (OGD)-induced mitochondrial oxidative damage by punctate-mitochondriadependent autophagy (Chen et al. 2017). But the relationship between FA-mediated anti-inflammatory effects and autophagy induction has not been clearly elucidated.

In this study, we used a cell inflammatory model to investigate the anti-inflammatory activity of ferulic acid and its related mechanism.

\section{Materials and methods}

\section{Reagents}

RPMI-1640 culture medium and foetal bovine serum were obtained from Gibco. Ferulic acid (FA), lipopolysaccharide (LPS), Phorbol-12-myristate-13-acetate (PMA), thiazolyl blue tetrazolium bromide (MTT), and 3-methyladenine (3-MA) were purchased from Sigma-Aldrich. MCC950 was purchased from Abmole Bioscience Inc. DCFH-DA was purchased from Beyotime Biotechnology Co., Ltd. The reverse transcription kits and SYBR Premix Ex Taq were purchased from Takara Biomedical Technology. The antibodies against Microtubule Associated Protein 1 Light Chain 3 Beta (LC3), Sequestosome 1 (p62), Beclin-1, NLRP3, pro-caspase-1, caspase-1, pro-Interleukin 1 Beta (IL-1 $1 \beta$ ), IL- $1 \beta$ and Glyceraldehyde-3-Phosphate Dehydrogenase (GAPDH) were purchased from ABclonal Technology. The Human IL-6 ELISA Kit, Human IL- $1 \beta$ ELISA kit and human TNF- $\alpha$ ELISA Kit were purchased from Multisciences (Lianke) Biotech, Co., Ltd. All other reagents used were obtained from local commercial sources.

\section{THP-1 cell differentiation}

The THP-1 (human monocyte-macrophage cell line; ATCC) was cultured in RPMI-1640 medium supplemented with $10 \%$ foetal calf serum, HEPES $(10 \mathrm{mM})$ and $\beta$-mercaptoethanol $(0.05 \mathrm{mM})$ at $37{ }^{\circ} \mathrm{C}$ in a $\mathrm{CO}_{2}$ incubator. The THP- 1 cells were distributed into cell cultured plate (at a concentration of $2 \times 10^{5}$ cells $/ \mathrm{mL}$ ) and cultured with $25 \mathrm{nM}$ PMA for $48 \mathrm{~h}$ (Lund et al. 2016). After discarding the medium containing PMA, the THP-1-derived macrophages $(\mathrm{M} \varphi \mathrm{s})$ were first 
treated with FA $(5,10,20 \mu \mathrm{M})$ or remained untreated for $24 \mathrm{~h}$ and then cultured with $100 \mathrm{ng} / \mathrm{mL}$ LPS for $4 \mathrm{~h}\left(37^{\circ} \mathrm{C}\right.$ and $\left.5 \% \mathrm{CO}_{2}\right)$. Then, the cell viability, expression of IL-6, IL- $1 \beta$, and TNF- $\alpha$ and ROS production were determined.

\section{MTT assay}

The THP-1-derived macrophages were induced differentiation and maintained as described above; then, the culture medium was removed, and $100 \mu \mathrm{L}$ of MTT solution $(2 \mathrm{mg} /$ $\mathrm{mL}$ ) were added per well for $4 \mathrm{~h}$ to allow MTT uptake. Subsequently, the excess MTT was discarded, and $100 \mu \mathrm{L}$ of dimethyl sulfoxide were added to dissolve the formed formazan. The absorbance was measured on a Thermo Scientific Microplate Reader with wavelengths of $490 \mathrm{~nm}$.

\section{Enzyme-linked immunosorbent assay (ELISA)}

After treating macrophages with ferulic acid and LPS as described above, the culture supernatants were collected and the amounts of TNF- $\alpha$, IL- 6 and IL- $1 \beta$ in the supernatant were measured using human ELISA kits in accordance with the manufacturer's use instructions.

\section{Real-time quantitative RT-PCR (RT-qPCR)}

The THP-1-derived macrophages were cultured $\left(1 \times 10^{6}\right.$ cells/well) as described above. The cells were treated with $100 \mathrm{ng} / \mathrm{mL}$ LPS and ferulic acid or not (negative control). Then, the culture medium was discarded from the culture plate wells, and the cells were washed twice with PBS. The cell total RNA was strictly isolated using TRIzol reagent and the A260/A280 ratio of the RNA samples were determined using a spectrophotometer. Then, the RNA $(2 \mu \mathrm{g})$ was converted to cDNA $(20 \mu \mathrm{L})$ using a PrimeScriptTM RT system, and qPCR was performed in triplicate using each cDNA sample for each gene. The following primers were used:

TNF- $\alpha$ forward primer (5'-3'): GAGGCCAAGCCCTGG TATG, TNF- $\alpha$ reverse primer ( $\left.5^{\prime}-3^{\prime}\right)$ : CGGGCCGATTGA TCTCAGC;

IL-6 forward primer $\left(5^{\prime}-3^{\prime}\right)$ : ACTCACCTCTTCAGA ACGAATTG, IL-6 reverse primer (5'-3'): CCATCTTTG GAAGGTTCAGGTTG;

IL-1 $\beta$ forward primer $\left(5^{\prime}-3^{\prime}\right)$ : ATGATGGCTTATTAC AGTGGCAA, IL-1 $\beta$ reverse primer (5'-3'): GTCGGAGAT TCGTAGCTGGA; and

GAPDH forward primer (5'-3'): AATGGAAATCCCATC ACCATCT, GAPDH reverse primer (5'-3'): CGCCCCACT TGATTTTGG.

The threshold cycle (CT) values were calculated and housekeeping gene is Gapdh. The relative gene expression values were calculated by the following formula: relative gene expression (treatment group/control group) $=2^{-\Delta \Delta \mathrm{CT}}$, where $\Delta \Delta \mathrm{CT}=(\mathrm{CT}$ target gene-CT Gapdh gene) treatment group - (CT target gene-CT Gapdh gene) control group. At least three biological samples were tested for per gene or treatment and a melt curve analysis was used to evaluate the specificity of the primers.

\section{Cell apoptosis and ROS content were determined by flow cytometry}

Cells in each treatment group were collected and washed with PBS. Cells were resuspended with $500 \mu \mathrm{L} 1 \times$ binding buffer, added Annexin V-FITC $(5 \mu \mathrm{L}) / \mathrm{PI}(10 \mu \mathrm{L})$ and incubated in dark for 10 min before analysing by flow cytometry.

DCFH-DA is a cellular permeable probe for the detection of intracellular reactive oxygen species (ROS). DCFH-DA was diluted to a suitable concentration $(10 \mu \mathrm{M})$ with serumfree medium. Then, the medium in the culture plate was discarded and an appropriate volume of DCFH-DA was added to cover the cells. Usually, no less than $1 \mathrm{~mL}$ of DCFH-DA was added to one well (six-well plate). The cells were incubated in an incubator at $37{ }^{\circ} \mathrm{C}$ for $20 \mathrm{~min}$. The cells were washed twice with serum-free medium three times and LPS $(100 \mathrm{ng} / \mathrm{mL})$ was added to the cell culture supernatant with or without the ferulic acid pretreatment. After $1 \mathrm{~h}$, the cells were collected and the fluorescence intensity was detected by flow cytometry.

\section{Western blot}

The treated macrophages were washed once with ice-cold PBS and lysed with RIPA lysis buffer supplemented with protease inhibitors. Then, the cell lysates were collected and centrifuged for $10 \mathrm{~min}$ at $13,000 \mathrm{rpm}$ at $4{ }^{\circ} \mathrm{C}$ to clear cellular debris deposits, and the protein concentrations were measured with a BCA protein assay. The samples were boiled at $100{ }^{\circ} \mathrm{C}$ for $10 \mathrm{~min}$ in loading buffer before the electrophoretic separation. The cell lysates were separated using $8-12 \%$ SDS-PAGE and then transferred onto PVDF membranes. After blocking the membranes with 5\% nonfat milk in TBST at room temperature for $2 \mathrm{~h}$, the membranes were incubated with LC3, p62, Beclin-1, NLRP3, pro-caspase-1, caspase-1, pro-IL-1 $\beta$, IL-1 $\beta$ and GAPDH antibodies and corresponding secondary antibodies. Finally, the protein-antibody complex signals were detected by enhanced chemiluminescence using a commercial kit according to the manufacturer's suggested protocols. ImageJ software was used to quantitate the intensity of the bands.

\section{Data analysis}

All results are presented as the means \pm standard deviation. In the statistical analysis, one-way analysis of variance (one-way ANOVA) was performed using GraphPad Prism 6.0 software. A significant difference was defined as $P<0.05$. 


\section{Results}

\section{The effect of ferulic acid on the activity of THP-1-derived macrophages}

To determine whether monocytes differentiate into macrophages, we continuously observed the changes in cell morphology through microscopy. As shown in Fig. 1A, as the PMA induction time increased, the THP-1 cells changed from an elliptical suspension state to an irregular attachment state with a large number of pseudopods, indicating that the THP-1 cells had been successfully induced into macrophages (Lund et al. 2016). To further clarify the cytotoxic effect of ferulic acid, the THP-1-derived macrophages were exposed to different concentrations of FA $(5,10,20 \mu \mathrm{M})$ for $24 \mathrm{~h}$. Compared with the control group, low, medium, and high concentration ferulic acid groups have decreased cell viability and increased apoptosis rate in a dose-dependent manner $(P<0.05)$ (Fig. 1, Table 1).

\section{FA suppresses LPS-induced TNF- $\alpha$, IL-6 and IL-1 $\beta$ expression and ROS production in vitro}

TNF- $\alpha$, IL- 6 and IL- $1 \beta$ are major inflammatory mediators contributing to the pathogenesis of inflammatory diseases. THP-1-derived macrophages constitutively expressed lots of inflammatory cytokines, including TNF- $\alpha$, IL- 6 and IL- $1 \beta$, at low levels. To elucidate whether FA could regulate the expression of inflammatory mediators, the total mRNA extracted from the treated macrophages was subjected to
Table 1 Effects of ferulic acid on cell viability and apoptosis rate of THP-1-derived macrophages $(\bar{x} \pm s)$

\begin{tabular}{lllc}
\hline Group & $\begin{array}{l}\text { Concentration } \\
\left(\mu \mathrm{mol} \mathrm{mL} \mathrm{mL}^{-1}\right)\end{array}$ & Cell viability (\%) & Apoptosis rate (\%) \\
\hline Blank control & 0 & $98.63 \pm 5.07$ & $2.63 \pm 0.28$ \\
FA-L & 5 & $96.96 \pm 1.77$ & $2.93 \pm 0.12$ \\
FA-M & 10 & $90.20 \pm 2.24$ & $6.10 \pm 0.96^{*}$ \\
FA-H & 20 & $82.41 \pm 3.30^{*}$ & $11.50 \pm 1.02^{* *}$ \\
\hline
\end{tabular}

$L$ low concentration; $M$ medium concentration; $H$ high concentrations Compared with blank control group, $* P<0.05$, $* * P<0.01$

a qPCR analysis. The experimental groups were pretreated with $20 \mu \mathrm{M}$ FA and then stimulated with LPS at $100 \mathrm{ng} / \mathrm{mL}$ for $4 \mathrm{~h}$. Non-stimulated cells were used as a negative control and LPS-stimulated cells were used as a positive control. As shown in Fig. 2, the addition of LPS led to a remarkably increase in the mRNA expression of TNF- $\alpha$, IL- 6 and IL- $1 \beta$, and the pretreatment with FA obviously decreased the mRNA expression of TNF- $\alpha$, IL- 6 and IL- $1 \beta$. We also detected the impact of FA on ROS formation and found that FA reduced ROS formation in macrophages activated by LPS in a dose-dependent manner (Table 2).

\section{FA-induced autophagy regulates inflammatory cytokine release}

Autophagy is a process that maintains cell homeostasis by degrading certain cell contents and has been confirmed to play a key role in inflammation, programmed cell death, and

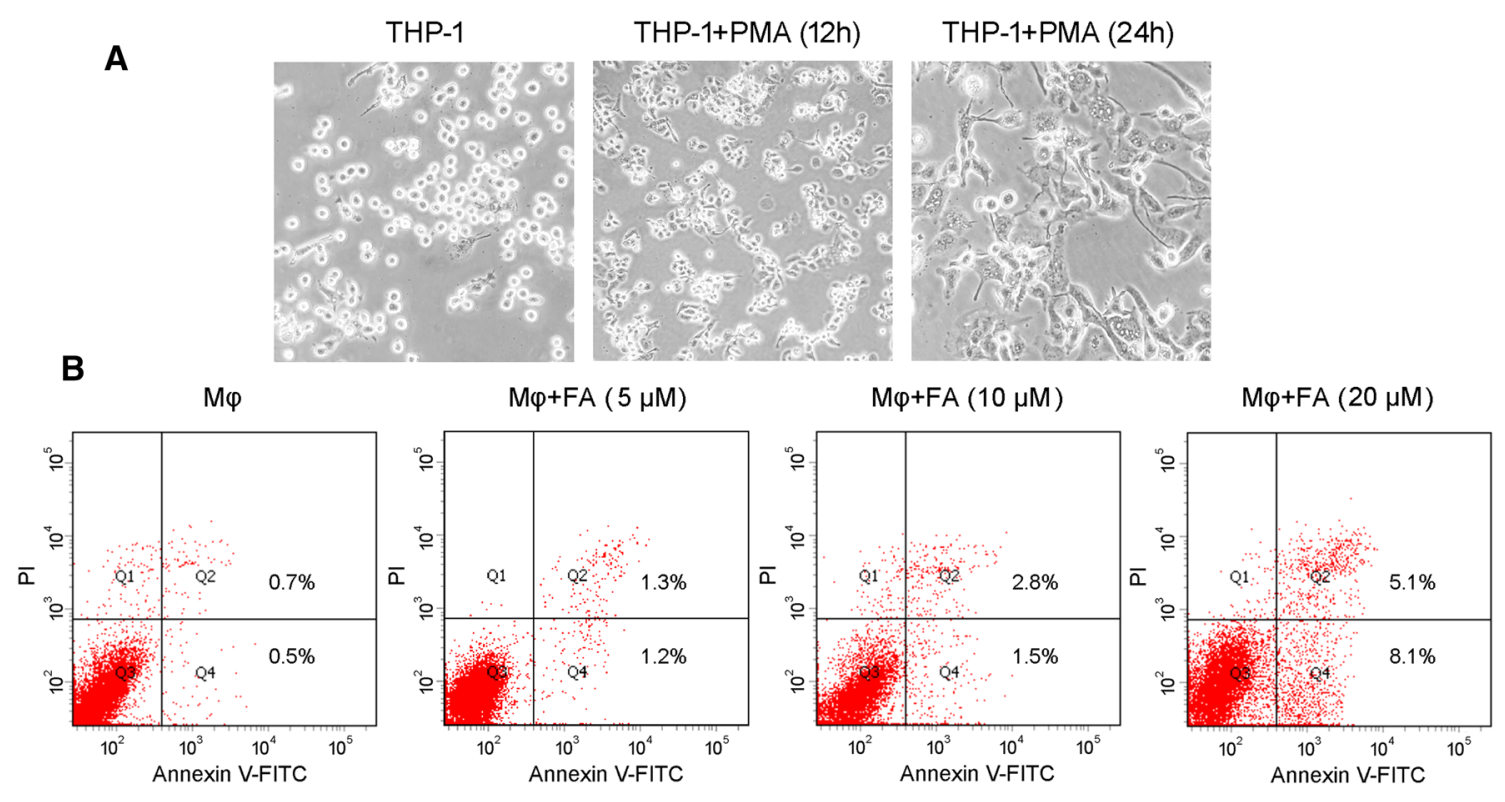

Fig. 1 A Morphology of THP-1-derived macrophages treated with PMA for 12 and $24 \mathrm{~h}$ as observed by microscopy (200×). B Flow cytometry was used to detect the apoptosis rate of THP-1-derived macrophages in each group. Values are means \pm SD of three independent experiments 


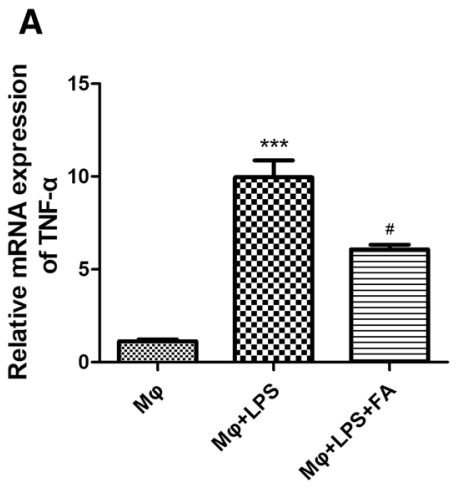

Fig. 2 FA suppressed LPS-induced proinflammatory cytokine release in vitro. A-C THP-1-derived macrophages were pretreated with FA $(20 \mu \mathrm{M})$ for $24 \mathrm{~h}$ before stimulation with LPS $(100 \mathrm{ng} / \mathrm{mL})$ for another $4 \mathrm{~h}$, and the proinflammatory cytokines $T N F-\alpha, I L-6$ and $I L$ -

Table 2 Effect of ferulic acid on the expression of ROS in THP-1-derived macrophages $(\bar{x} \pm s)$

\begin{tabular}{llc}
\hline Group & $\begin{array}{l}\text { Concentration } \\
\left(\mu \mathrm{mol} \mathrm{mL} \mathrm{mL}^{-1}\right)\end{array}$ & $\begin{array}{l}\text { Relative DCF } \\
\text { fluorescence }(\%)\end{array}$ \\
\hline Blank control & - & $98.00 \pm 1.16$ \\
Model & - & $125.0 \pm 2.89^{* * * *}$ \\
FA-L & 5 & $119.7 \pm 2.60$ \\
FA-M & 10 & $102.3 \pm 1.45^{\# \#}$ \\
FA-H & 20 & $102.3 \pm 3.71^{\# \#}$ \\
\hline
\end{tabular}

Compared with blank control group, $* * * P<0.001$; Compared with model group, ${ }^{\# \#} P<0.01$

adaptive immunity (Glick et al. 2010). To further clarify the possible relationship between autophagy and inflammation, we detected the expression levels of autophagy-related proteins in treated cells. As shown in Figs. 3B and 4A, no significant difference could be observed in the expression ratio of LC3, p62 and Beclin-1 between the LPS exposure group and the control group. However, the protein expression levels of LC3-II and Beclin-1 were significantly increased in the FA-pretreated cells, indicating the occurrence of autophagy. To further elucidate the effect of autophagy on inflammation, 3-MA (an autophagy inhibitor) was applied. As demonstrated in Figs. 3 and 4A, 3-MA obviously decreased LC3II and Beclin-1 expression. Moreover, 3-MA reversed the reduction in inflammatory factor (TNF- $\alpha$, IL- 6 and IL-1 $\beta$ ) release induced by FA, suggesting that autophagy plays a vital role in regulating inflammation (Fig. 4C).

\section{FA inhibits LPS-induced activation of the NLRP3 inflammasome}

NLRP3 inflammasome, an intracellular signaling platform that induces the activation of inflammatory caspases and the release of interleukin- $1 \beta$, is an important part of innate

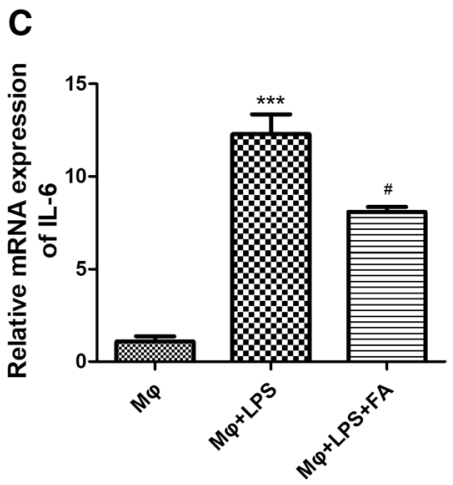

$1 \beta$ were measured using RT-qPCR. Values are the means \pm SD of three independent experiments. $* * P<0.01$, $* * * P<0.001$, compared with the control group; ${ }^{\#} P<0.05$, compared with the LPS-treated group

immunity (Zhao and Zhao 2020). To further clarify the effect of FA on inflammasome activation, we examined the protein expression of the inflammasome signalling pathway. As expected, the protein expression levels of NLRP3, activated caspase- 1 and IL- $1 \beta$ were significantly increased after the LPS induction. However, the high level of IL-1 $\beta$ induced by LPS (Fig. 5A, $P<0.01$ ) was dramatically decreased in the $20 \mu \mathrm{M}$ FA pretreatment group. Moreover, the increased expression of NLRP3 and caspase-1 triggered by LPS was also attenuated in the FA pretreatment group. These results strongly confirmed that FA inhibited the LPS-triggered activation of the NLRP3 inflammasome in the THP-1-derived macrophages.

\section{FA-mediated inhibition of NLRP3 inflammasome activation is mediated by autophagy induction}

To further explore the role of inflammasome in the production of inflammatory factors, we used the NLRP3 inflammasome inhibitor MCC950. As illustrated in Fig. 6, the increased levels of NLRP3, activated caspase-1 and IL-1 $\beta$ induced by LPS were attenuated dramatically in the MCC950 pretreatment group (Fig. 6A, B, $P<0.01$ ). Moreover, a marked decrease in the release of TNF- $\alpha$, IL- 6 and IL-1 $\beta$ expression was detected (Fig. $6 \mathrm{C}-\mathrm{E}, P<0.01$ ).

This research found that FA reduced the expression of inflammatory factors by inducing autophagy. We also found that FA inhibits LPS-induced activation of the NLRP3 inflammasome. To further clarify the relationship between autophagy and the NLRP3 inflammasome in LPS-treated THP-1-derived macrophages, we used the autophagy inhibitor 3-MA and detected the protein expression of NLRP3 inflammasome-related proteins. As illustrated in Fig. 6A, B, the protein expression of NLRP3, activated caspase- 1 and IL- $1 \beta$ was markedly decreased in the FA-treated cells, and 3-MA markedly increased NLRP3, activated caspase-1 and 


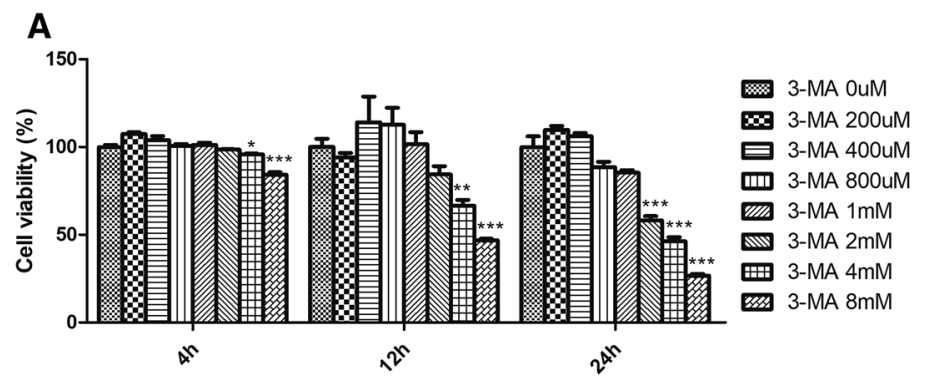

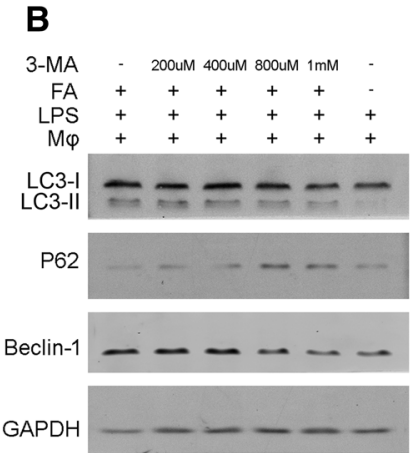

Fig. 3 FA induces autophagy and 3-MA inhibits FA-induced autophagy. A Effect of different concentration of 3-MA on cell activity. THP-1-derived macrophages were treated with 3-MA $(0,200 \mu \mathrm{M}$, $400 \mu \mathrm{M}, 800 \mu \mathrm{M}, 1 \mathrm{mM}, 2 \mathrm{mM}, 4 \mathrm{mM}, 8 \mathrm{mM})$ for 4,12 and $24 \mathrm{~h}$ and cell activity was detected by MTT assay. $* P<0.05$, $* * P<0.01$, $* * * P<0.001$, compared with the control group (3-MA $0 \mu \mathrm{M}$ group). B, C 3-MA inhibits autophagy of THP-1-derived macrophages. THP1-derived macrophages were treated with LPS $(100 \mathrm{ng} / \mathrm{mL})$ for $4 \mathrm{~h}$ in

IL-1 $\beta$ protein expression (Fig. 6A, B, $P<0.01$ ). To sum up, the data show that FA may downregulate the expression of inflammatory factors by inducing autophagy and inhibiting the activation of the NLRP3 inflammasome.

\section{Discussion}

Inflammation is an important defense response of the innate immune system against various injurious factors (Medzhitov 2010). During inflammation, a series of local reactions centered on the vascular system limited and eliminated injury factors, promoted the healing of damaged tissue and involves a network of cellular responses and cytokines, including IL-1 $\beta$, IL-6, and TNF- $\alpha$ (Medzhitov 2008). Generally speaking, the release of these inflammatory mediators is beneficial to the body, but excessive release of inflammatory mediators is harmful to the body, and is even closely related to a variety of inflammation-related diseases (Back et al. 2019; Mahmudpour et al. 2020). IL-6 is an important inflammatory mediator with a pleiotropic effect on cancer progression, inflammation and immune response (Tanaka et al. 2014). IL-6 is mainly synthesized and secreted by immune cells ( $\mathrm{T}$ cells, monocytes macrophages) and non-immune cells the presence or absence of pretreatment with FA $(20 \mu \mathrm{M})$ for $24 \mathrm{~h}$ and 3-MA $(0,200 \mu \mathrm{M}, 400 \mu \mathrm{M}, 800 \mu \mathrm{M}, 1 \mathrm{mM})$ for $4 \mathrm{~h}$. The protein levels of LC3, p62, and Beclin-1 were evaluated by a Western blot analysis. GAPDH was used as a loading control. $* P<0.05$, $* * P<0.01$, $* * * P<0.001$, compared with the LPS-treated group; ${ }^{\wedge} P<0.05$, ${ }^{\wedge} P<0.01,{ }^{\wedge \wedge} P<0.001$, compared with the LPS + FA-treated group; $n s$ no significant difference

(fibroblasts, endothelial cells) during infection and tissue injury (Mihara et al. 2012). Interleukin- $1 \beta$ is a vital mediator of inflammatory response (Gabay et al. 2010; LopezCastejon and Brough 2011). Tumor necrosis factor- $\alpha$ (TNF$\alpha)$ is an inflammatory mediator produced by mononuclear macrophages during inflammation (Atzeni et al. 2020; Karki et al. 2021). This cytokine is not only involved in multiple intracellular signaling events leading to necrosis or apoptosis, but also important in the fight against infection and cancer (Atzeni et al. 2020; Karki et al. 2021). In this study, the addition of LPS led to a remarkably increase in the mRNA expression of TNF- $\alpha$, IL- 6 and IL- $1 \beta$, and the pretreatment with FA obviously decreased the mRNA expression of TNF$\alpha$, IL- 6 and IL- $1 \beta$.

Ferulic acid is a kind of widely distributed phenolic acid, which is often cross-linked with lignin and polysaccharide to form part of the cell wall of plants (de Oliveira et al. 2015). It was found that ferulic acid had strong antioxidant activity, and could scavenging hydrogen peroxide, hydroxyl radical and superoxide radical (Zhao and Moghadasian 2008). At the same time, ferulic acid has also been proved to have antibacterial and anti-inflammatory effects (Zhao and Moghadasian 2008). FA also has been confirmed to be a promising substance protecting against high-fat diet-induced weight 


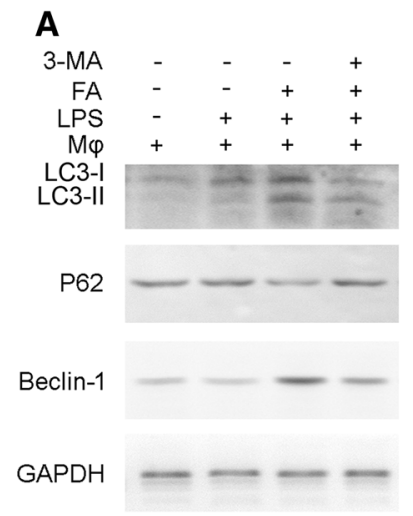

B

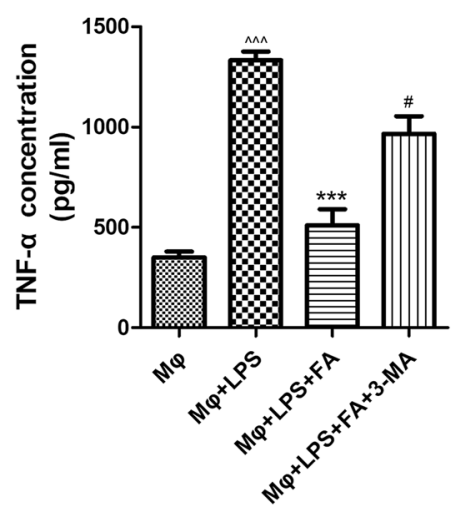

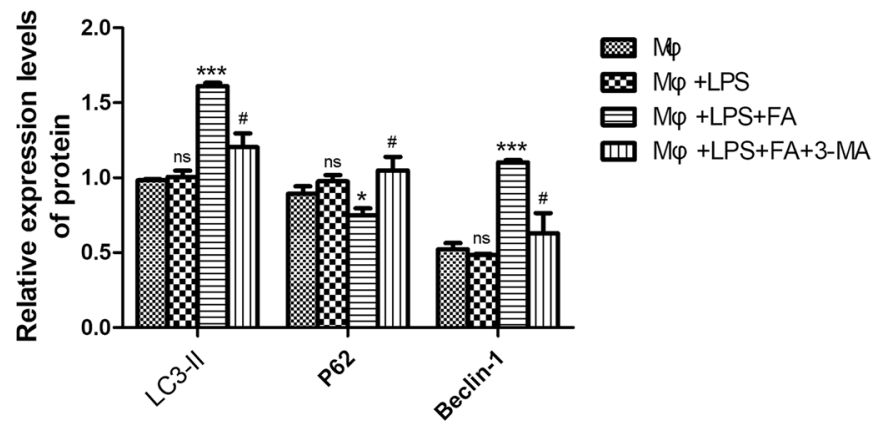

C

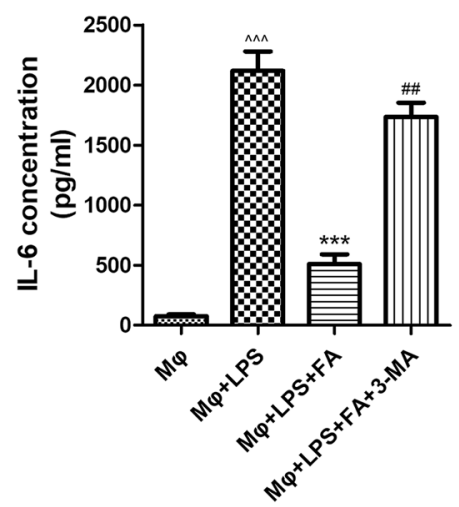

D

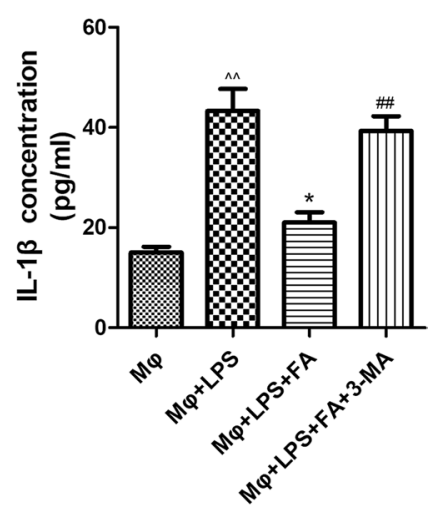

Fig. 4 FA inhibited inflammation by enhancing autophagy. A, B THP-1-derived macrophages were pretreated with FA $(20 \mu \mathrm{M})$ or 3-MA ( $1 \mathrm{mM})$ before stimulation with LPS $(100 \mathrm{ng} / \mathrm{mL})$ for another $4 \mathrm{~h}$, and LC3, p62, and Beclin-1 protein expression was detected by a Western blot analysis. C-E The cytokines TNF- $\alpha$, IL-6, and IL-1 $\beta$ were determined using ELISA kits. Values are the means \pm SD of three independent experiments. ${ }^{\wedge} P P<0.01,{ }^{\wedge} \wedge \wedge P<0.001$, compared with the control group; $* P<0.05$, *** $P<0.001$, compared with the LPS-treated group; ${ }^{\#} P<0.05$, ${ }^{\# \#} P<0.01$, compared with the LPS + FA-treated group

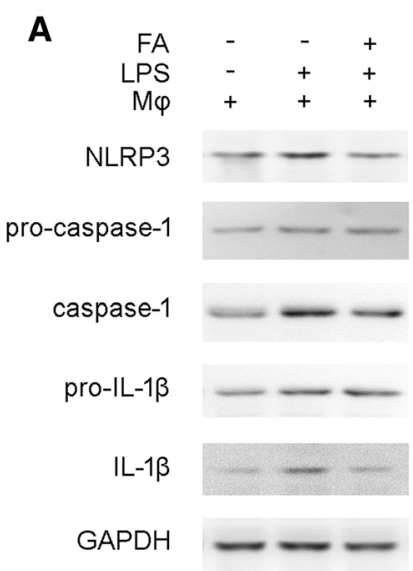

Fig. 5 LPS-induced activation of the NLRP3 inflammasome in THP-1-derived macrophages is attenuated by FA treatment. A THP-1-derived macrophages were treated with LPS $(100 \mathrm{ng} / \mathrm{mL})$ for $4 \mathrm{~h}$ in the presence or absence of pretreatment with FA $(20 \mu \mathrm{M})$ for $24 \mathrm{~h}$. The protein levels of NLRP3, pro-caspase-1, caspase-1, pro-IL-1 $\beta$ and IL-1 $\beta$ were evaluated by a Western blot analysis.

B

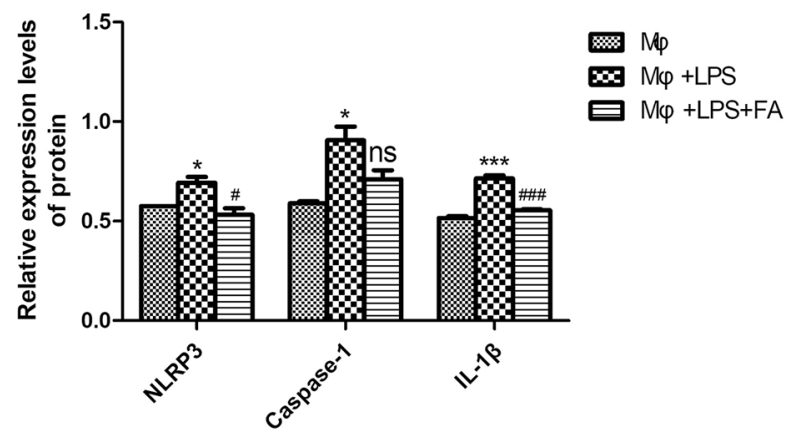

GAPDH was used as a loading control. B Densitometry analysis of the effects of FA on the expression of NLRP3, caspase-1 and IL-1 $\beta$. Values are the means \pm SD of three independent experiments. $* P<0.05,{ }^{*} * *<0.001$, compared with the control group; ${ }^{\#} P<0.05$, ${ }^{\# \# \#} P<0.001$, compared with the LPS-treated group; $n s$ no significant difference 
A

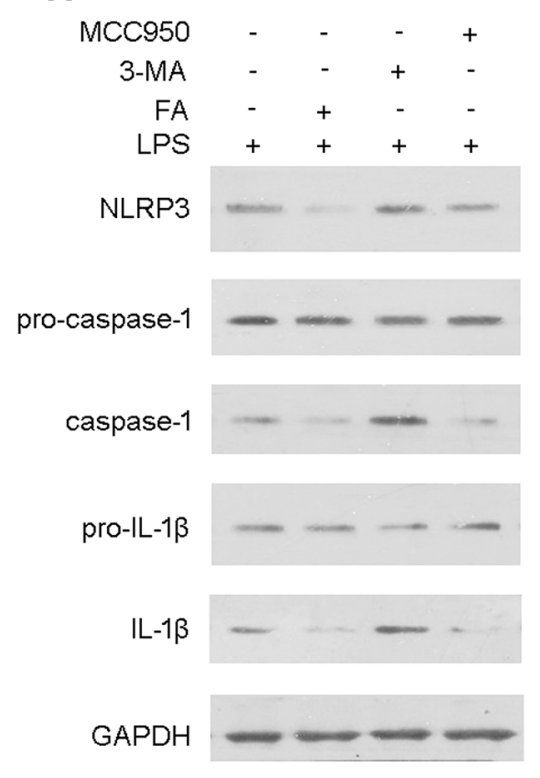

C

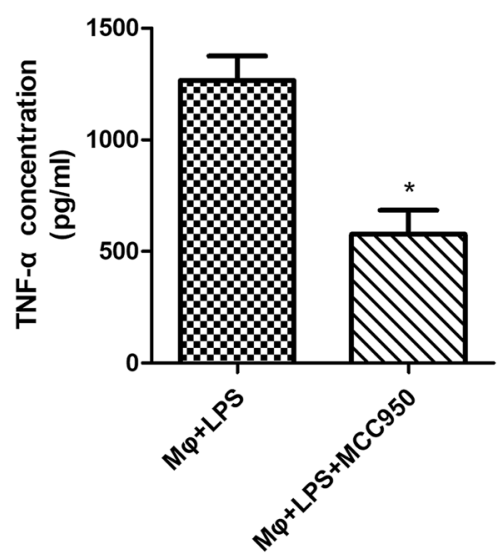

B

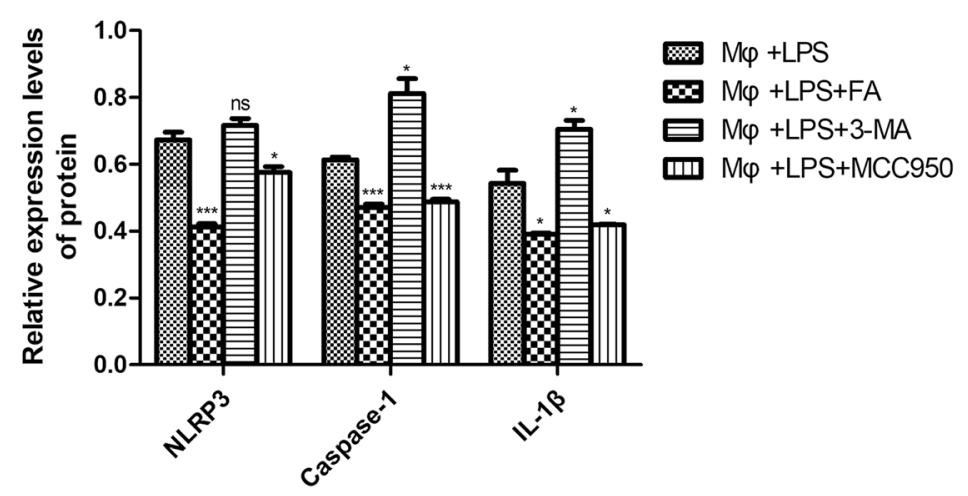

D

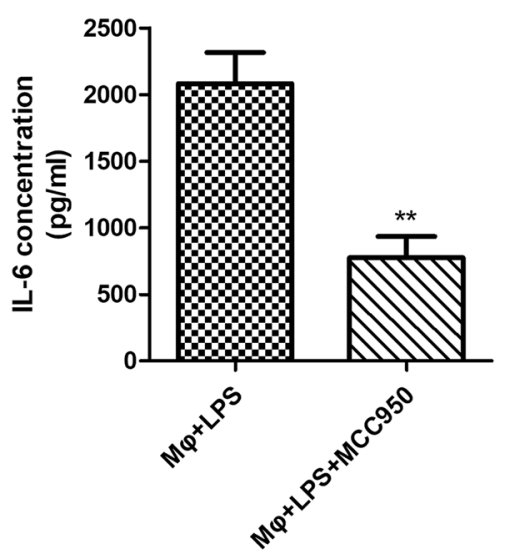

E

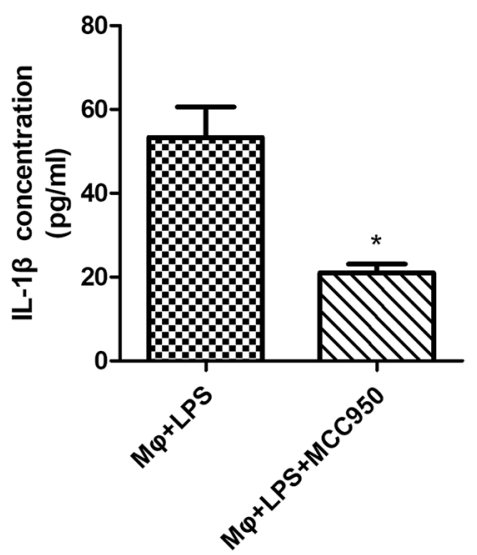

Fig. 6 Autophagy regulated the activation of the NLRP3 inflammasome in THP-1-derived macrophages. A THP-1-derived macrophages were treated with LPS $(100 \mathrm{ng} / \mathrm{mL})$ for $4 \mathrm{~h}$ in the presence or absence of pretreatment with FA $(20 \mu \mathrm{M})$ for $24 \mathrm{~h}$ and 3-MA $(1 \mathrm{mM})$ or MCC950 $(10 \mu \mathrm{M})$ for $4 \mathrm{~h}$. The protein levels of NLRP3, pro-caspase-1, caspase- 1 , pro-IL- $1 \beta$ and IL- $1 \beta$ were evaluated by a Western blot analysis. GAPDH was used as a loading control. B Densitometry analysis of the effects of FA, 3-MA and MCC950 on the expression of NLRP3, pro-caspase-1, caspase-1, pro-IL-1 $\beta$ and IL-1 $\beta$. C-E The effect of MCC950 on the expression of TNF- $\alpha$, IL- 6 and IL- $1 \beta$ was examined by ELISA. Values are the means \pm SD of three independent experiments. $\mathbf{B} * P<0.05, * * * P<0.001$, compared with the control group; $\mathbf{C}-\mathbf{E} * P<0.05, * * P<0.01$, compared with the LPS-treated group; $n s$ no significant difference gain and Alzheimer's disease (Kesh et al. 2013; Meng et al. 2018). Our studies demonstrated that FA disturbed the proinflammatory activity of macrophages by decreasing TNF- $\alpha$, IL-6 and IL- $1 \beta$ secretion induced by LPS.

The inflammasomes are a class of macromolecular complexes that exist in the cytoplasm and are capable of sensing pathogenic microorganism infection, tissue injury and metabolic imbalance (Broz and Dixit 2016; Guo et al. 2015). NLRP3 inflammasome is the most widely studied inflammasome, which is mainly composed of NLRP3, ASC (apoptosis-associated speck-like protein containing a CARD) and procaspase-1 (Broz and Dixit 2016; Zhao and Zhao 2020). When receptors perceive signals from pathogen infection, tissue damage, or metabolic imbalance, they recruit ASC and pro-caspase- 1 to form inflammasomes, which in turn activate caspase- 1 and induced the secretion of IL-1 $\beta$ and IL-18 (Zhao and Zhao 2020). At present, it is believed that the activation of inflammasome may be related to the occurrence of a variety of diseases, and further study of inflammasome may provide a new theoretical basis for the prevention and treatment of related diseases (Guo et al. 2015). In this study, it was found that FA down-regulated the protein expression 


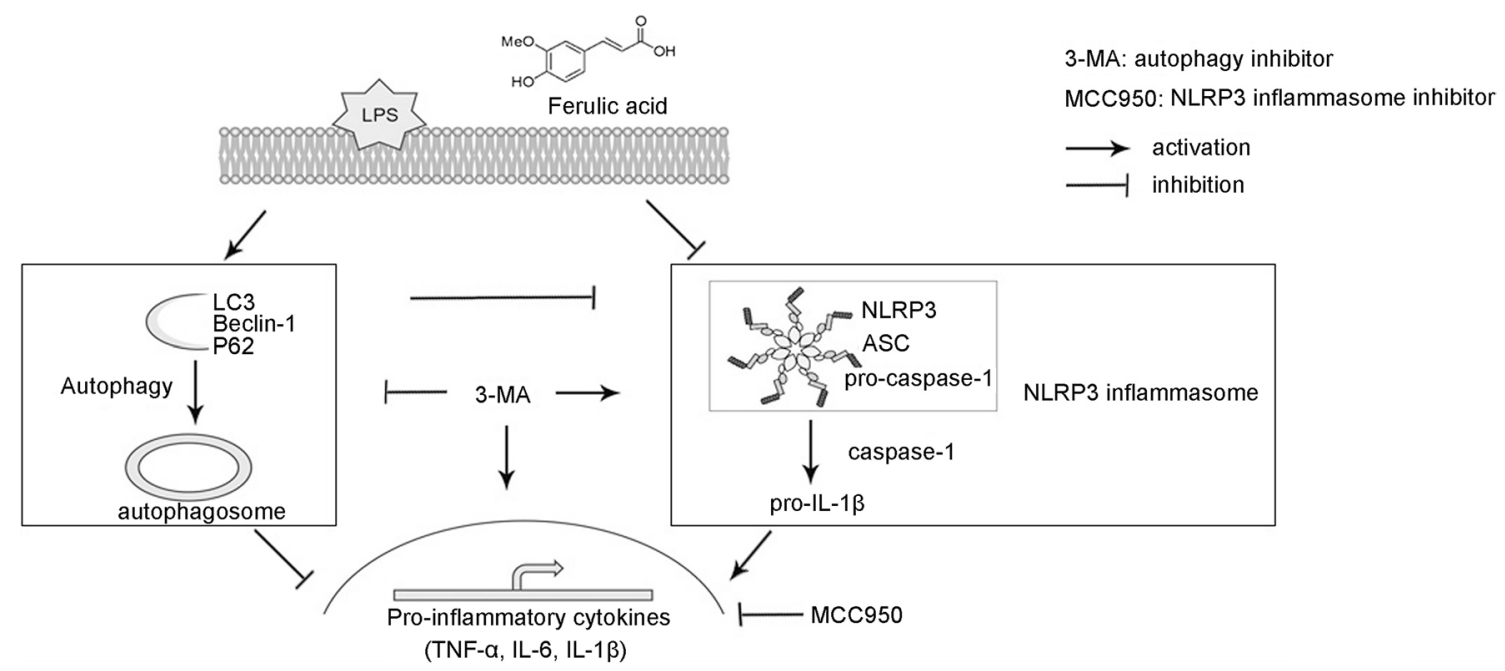

Fig. 7 Proposed model of the mechanism by which FA exerts antiinflammatory activity. FA can play an anti-inflammatory role by inducing autophagy and inhibiting the activation of inflammasome, thus inhibiting the release of inflammatory factors. Treatment of cells with the autophagy inhibitor 3-MA resulted in down-regulation of

of NLRP3, activated caspase- 1 and IL- $1 \beta$ and inhibited the LPS-triggered activation of the NLRP3 inflammasome. Meanwhile, when MCC950 was used to inhibit the activation of NLRP3 inflammasomes, the release of TNF- $\alpha$, IL- 6 and IL-1 $\beta$ was significantly reduced. These results suggest that FA may downregulate the expression of TNF- $\alpha$, IL- 6 and IL-1 $\beta$ by inhibiting the activation of NLRP3 inflammasomes.

Reports have indicated that NLRP3 inflammasome activation needs a second signal delivered from ROS production, potassium efflux or calcium influx (Franchi et al. 2012). To explore whether FA modulates the second signal required for NLRP3 activation, FA-pretreated THP-1-derived macrophages were stimulated with LPS, and then, ROS production was detected. The results showed that the FA pretreatment reduced LPS-induced intracellular ROS production (Fig. 2D). These results suggest that FA downregulates the activation of NLRP3 inflammasomes by inhibiting the expression of ROS.

Autophagy is a biochemical process that takes place in cells all the time (Glick et al. 2010). Its function is to encapsulate damaged organelles and misfolded proteins through the formation of autophagosomes and transport them to lysosomes for degradation (Glick et al. 2010; Parzych and Klionsky 2014). Many molecules have been shown to be involved in autophagy, including LC3, Beclin-1 and p62 (Glick et al. 2010). In this study, the effect of FA on macrophage autophagy was explored, and FA-induced autophagy by increasing LC 3 and Beclin-1 expression and decreasing p62 expression (Fig. 4). To further clarify the relationship between autophagy and autophagy, activation of inflammasome and increased expression of inflammatory factors. Treatment of the cells with the inflammasome activator inhibitor MCC950 reduced the expression of inflammatory cytokines in the cells

inflammation, 3-MA was selected to block autophagy. In this study, to further determine the appropriate concentration of 3-MA, the THP-1-derived macrophages were exposed to different concentrations of 3-MA $(0,200 \mu \mathrm{M}$, $400 \mu \mathrm{M}, 800 \mu \mathrm{M}, 1 \mathrm{mM}, 2 \mathrm{mM}, 4 \mathrm{mM}, 8 \mathrm{mM})$ for 4,12 and $24 \mathrm{~h}$. Compared with the control group, 2, 4 and $8 \mathrm{mM}$ 3-MA groups have significantly decreased cell viability $(P<0.05)$ (Fig. 3A). Therefore, in subsequent studies, concentrations of $0,200 \mu \mathrm{M}, 400 \mu \mathrm{M}, 800 \mu \mathrm{M}$ and $1 \mathrm{mM}$ were selected. To further clarify the effect of 3-MA on autophagic protein expression, we detected the expression levels of autophagy-related proteins in treated cells. As shown in Fig. 3B, with the increase of 3-MA concentration $(0,200 \mu \mathrm{M}, 400 \mu \mathrm{M}, 800 \mu \mathrm{M}, 1 \mathrm{mM}), \mathrm{LC} 3-\mathrm{II}$ and Beclin-1 protein expression level decreased significantly. Considering the cytotoxic effect and inhibition efficiency of autophagy, $800 \mu \mathrm{M}$ or $1 \mathrm{mM}$ may be a reasonable concentration of 3-MA. More importantly, the autophagy inhibitor 3-MA activated the NLRP3 inflammasome pathway and enhanced TNF- $\alpha$, IL- 6 and IL- $1 \beta$ release (Fig. 6 ). It is suggested that FA, on one hand, inhibits the expression of ROS and on the other hand, induces autophagy to inhibit the activation of inflammasome, and ultimately downregulates the expression and release of TNF- $\alpha$, IL- 6 and IL- $1 \beta$.

In conclusion, our study suggested that FA enhanced autophagy, inhibited the activation of NLRP3 inflammasome and reduced the expression and release of inflammatory factors. Our study provides evidence that FA might be a potential therapeutic agent for inflammatory diseases by virtue of its anti-inflammatory activity (Fig. 7). 
Acknowledgements This study was supported by Natural Science Foundation of Hubei Province (No. 2018CFB254), Wuhan COVID-19 Emergency Research Project (No. EX20D04), Natural Science Foundation of Jiangsu Province (No. BK20180269).

Author contributions YL, LS, WQ were responsible for designing research studies, conducting experiments and acquiring data. YL and YS were responsible for providing reagents, designing research studies, analyzing data, and writing the manuscript. All authors have read and approved the manuscript.

\section{Declarations}

Conflict of interest Yongjuan Liu declares she has no conflict of interest. Lu Shi declares she has no conflict of interest. Wenhong Qiu declares he has no conflict of interest. Yingying Shi declares she has no conflict of interest.

Ethical statement The study includes only laboratory studies on a cell line. No ethical or institutional approval was required.

\section{References}

Amic A, Markovic Z, Dimitric MJ et al (2020) Antioxidative potential of ferulic acid phenoxyl radical. Phytochemistry 170:112218

Atzeni F, Nucera V, Gerratana E et al (2020) Concerns about the safety of anti-TNF agents when treating rheumatic diseases. Expert Opin Drug Saf 19(6):695-705

Back M, Yurdagul AJ, Tabas I et al (2019) Inflammation and its resolution in atherosclerosis: mediators and therapeutic opportunities. Nat Rev Cardiol 16(7):389-406

Biriken D, Yazihan N, Yilmaz S (2018) Investigation of cytokine and midkine responses of human THP-1 leukemia cells induced by phorbol-12-Myristate-13-Acetate (PMA) at different concentrations and times. Mikrobiyol Bul 52(2):147-155

Broz P, Dixit VM (2016) Inflammasomes: mechanism of assembly, regulation and signalling. Nat Rev Immunol 16(7):407-420

Cao Z, Wang Y, Long Z et al (2019) Interaction between autophagy and the NLRP3 inflammasome. Acta Biochim Biophys Sin (shanghai) 51(11):1087-1095

Chen JL, Duan WJ, Luo S et al (2017) Ferulic acid attenuates brain microvascular endothelial cells damage caused by oxygenglucose deprivation via punctate-mitochondria-dependent mitophagy. Brain Res 1666:17-26

de Oliveira DM, Finger-Teixeira A, Mota TR et al (2015) Ferulic acid: a key component in grass lignocellulose recalcitrance to hydrolysis. Plant Biotechnol J 13(9):1224-1232

DiDonato JA, Mercurio F, Karin M (2012) NF-kappaB and the link between inflammation and cancer. Immunol Rev 246(1):379-400

Dinarello CA (2011) Interleukin-1 in the pathogenesis and treatment of inflammatory diseases. Blood 117(14):3720-3732

Franchi L, Munoz-Planillo R, Nunez G (2012) Sensing and reacting to microbes through the inflammasomes. Nat Immunol 13(4):325-332

Gabay C, Lamacchia C, Palmer G (2010) IL-1 pathways in inflammation and human diseases. Nat Rev Rheumatol 6(4):232-241

Glass CK, Saijo K, Winner B et al (2010) Mechanisms underlying inflammation in neurodegeneration. Cell 140(6):918-934

Glick D, Barth S, Macleod KF (2010) Autophagy: cellular and molecular mechanisms. J Pathol 221(1):3-12
Gruber EJ, Leifer CA (2020) Molecular regulation of TLR signaling in health and disease: mechano-regulation of macrophages and TLR signaling. Innate Immun 26(1):15-25

Guo H, Callaway JB, Ting JP (2015) Inflammasomes: mechanism of action, role in disease, and therapeutics. Nat Med 21(7):677-687

Karki R, Sharma BR, Tuladhar S et al (2021) Synergism of TNFalpha and IFN-gamma triggers inflammatory cell death, tissue damage, and mortality in SARS-CoV-2 infection and cytokine shock syndromes. Cell 184(1):149-168

Kesh SB, Sikder K, Manna K et al (2013) Promising role of ferulic acid, atorvastatin and their combination in ameliorating high fat diet-induced stress in mice. Life Sci 92(17-19):938-949

Li C, Chen L, Song M et al (2020) Ferulic acid protects cardiomyocytes from TNF-alpha/cycloheximide-induced apoptosis by regulating autophagy. Arch Pharm Res 43(8):863-874

Liu S, Huang L, Lin Z et al (2017) RhoB induces the production of proinflammatory cytokines in TLR-triggered macrophages. Mol Immunol 87:200-206

Lopez-Castejon G, Brough D (2011) Understanding the mechanism of IL-1beta secretion. Cytokine Growth Factor Rev 22(4):189-195

Lund ME, To J, O'Brien BA et al (2016) The choice of phorbol 12-myristate 13-acetate differentiation protocol influences the response of THP-1 macrophages to a pro-inflammatory stimulus. J Immunol Methods 430:64-70

Mahmudpour M, Roozbeh J, Keshavarz M et al (2020) COVID-19 cytokine storm: the anger of inflammation. Cytokine 133:155151

Medzhitov R (2008) Origin and physiological roles of inflammation. Nature 454(7203):428-435

Medzhitov R (2010) Inflammation 2010: new adventures of an old flame. Cell 140(6):771-776

Meng G, Meng X, Ma X et al (2018) Application of ferulic acid for Alzheimer's disease: combination of text mining and experimental validation. Front Neuroinform 12:31

Mihara M, Hashizume M, Yoshida H et al (2012) IL-6/IL-6 receptor system and its role in physiological and pathological conditions. Clin Sci (Lond) 122(4):143-159

Parzych KR, Klionsky DJ (2014) An overview of autophagy: morphology, mechanism, and regulation. Antioxid Redox Signal 20(3):460-473

Petes C, Wynick C, Guzzo C et al (2017) IL-27 enhances LPS-induced IL-1beta in human monocytes and murine macrophages. J Leukoc Biol 102(1):83-94

Tanaka T, Narazaki M, Kishimoto T (2014) IL-6 in inflammation, immunity, and disease. Cold Spring Harb Perspect Biol 6(10):a16295

Tang YS, Zhao YH, Zhong Y et al (2019) Neferine inhibits LPS-ATPinduced endothelial cell pyroptosis via regulation of ROS/NLRP3/ Caspase-1 signaling pathway. Inflamm Res 68(9):727-738

Wu J, Xue X, Fan G et al (2021) Ferulic acid ameliorates hepatic inflammation and fibrotic liver injury by inhibiting PTP1B activity and subsequent promoting AMPK phosphorylation. Front Pharmacol 12:754976

Xu T, Song Q, Zhou L et al (2021) Ferulic acid alleviates lipotoxicity-induced hepatocellular death through the SIRT1-regulated autophagy pathway and independently of AMPK and Akt in AML-12 hepatocytes. Nutr Metab (Lond) 18(1):13

Zhang SH, Liu D, Hu Q et al (2019) Ferulic acid ameliorates pentylenetetrazol-induced seizures by reducing neuron cell death. Epilepsy Res 156:106183

Zhao Z, Moghadasian MH (2008) Chemistry, natural sources, dietary intake and pharmacokinetic properties of ferulic acid: a review. Food Chem 109(4):691-702 
Zhao C, Zhao W (2020) NLRP3 inflammasome-a key player in antiviral responses. Front Immunol 11:211

Zhao D, Jiang Y, Sun J et al (2019) Elucidation of the anti-inflammatory effect of vanillin In Lps-activated THP-1 cells. J Food Sci 84(7):1920-1928

Zhou Z, Shi T, Hou J et al (2020) Ferulic acid alleviates atopic dermatitis-like symptoms in mice via its potent anti-inflammatory effect. Immunopharmacol Immunotoxicol 42(2):156-164
Publisher's Note Springer Nature remains neutral with regard to jurisdictional claims in published maps and institutional affiliations. 